Research Paper

\title{
Molecular Mechanism of Anti-Cancer Activity of the Nano-Drug C-PC/CMC-CD59sp NPs in Cervical Cancer
}

\author{
Liangqian Jiang ${ }^{1 *}$, Yujuan Wang1 ${ }^{*}$, Feng Zhu1 ${ }^{1}$, Guoxiang Liu1 ${ }^{1}$, Huihui Liu1 ${ }^{1}$, Huanhuan Ji1, \\ Shuhua Zheng², Bing $\mathrm{Li}^{1 凶}$ \\ 1. Department of Genetics and Cell Biology, Basic medical school, 308 Ningxia Road, Qingdao University, Qingdao, China, 266071 \\ 2. The Sheila and David Fuente Graduate Program in Cancer Biology, University of Miami Miller School of Medicine, Miami, FL, USA. \\ * Liangqian Jiang and Yujuan Wang contributed equally to this article. \\ $\triangle$ Corresponding author: Professor Bing Li, Department of Genetics and Cell Biology, Basic medical college, 308 Ningxia Road, Qingdao University, Qingdao, \\ China, 266071. Email: libing_516619@163.com \\ (C) Ivyspring International Publisher. This is an open access article distributed under the terms of the Creative Commons Attribution (CC BY-NC) license \\ (https://creativecommons.org/licenses/by-nc/4.0/). See http://ivyspring.com/terms for full terms and conditions.
}

Received: 2018.05.25; Accepted: 2018.10.21; Published: 2019.01.01

\begin{abstract}
The novel tumor targeted nano-drug C-PC/CMC-CD59sp nanoparticles were constructed with carbocymethyl chitosan (CMC), C-phycocyanin (C-PC) and CD59 specific ligand peptide (CD59sp). The anti-tumor drug mechanism of the C-PC/CMC-CD59sp NPs was further explored in cervical cancer cells (HeLa and $\mathrm{SiHa}$ ) in vitro and in vivo. We found that the C-PC/CMC-CD59sp NPs could inhibit the proliferation and induce G0/G1 cell cycle arrest in cervical cancer HeLa and SiHa cells, and the cell proliferation was reduced in a dose-dependent manner. We further found that the C-PC/CMC-CD59sp NPs regulated the cell cycle via up-regulating the expression of p21, and then down-regulating the expressions of Cyclin DI and CDK4 in vivo. Compared with C-PC and C-PC/CMC NPs, the pro-apoptosis effects of the C-PC/CMC-CD59sp NPs were more significant for HeLa and $\mathrm{SiHa}$ cells in vitro. Moreover, the C-PC/CMC-CD59sp NPs up-regulated the expression of cleaved caspase-3 and down-regulated the expression of bcl-2. In addition, compared with C-PC and C-PC/CMC, the C-PC/CMC-CD59sp NPs significantly inhibited MMP-2 protein expression in vivo. Our data suggested that the anti-tumor effects of C-PC/CMC-CD59sp NPs were better than C-PC and C-PC/CMC NPs. Our laboratory constructed a new drug delivery system and proved the effective antitumor effects of C-PC/CMC-CD59sp, which would widen the application of $\mathrm{C}-\mathrm{PC}$ as a potential anti cervical cancer drug.
\end{abstract}

Key words: C-PC/CMC-CD59sp, cell cycle arrest, apoptosis, Bcl-2/Cleaved caspase-3, cervical cancer

\section{Introduction}

Cervical cancer is the second most common cancer among females in less developed regions and countries, and it is the third major cause of cancer death among females in less developed countries [1]. In 2012, it was estimated that there were about 527,600 new cases of cervical cancer and 265,700 deaths of cervical cancer worldwide [1]. In the United States, cervical cancer is the second major cause of cancer death among females aged from 20 to 39, and just lower than breast cancer in 2014 [2]. The underdeveloped regions of the world, such as Latin America and the Caribbean, and sub-Saharan Africa and Melanesia, have a higher incidence of cervical cancer [1]. It is serious that nearly $90 \%$ deaths of cervical cancer occur in the less developed regions of the world [1]. Especially in Eastern, Middle, and Southern Africa, cervical cancer is the leading cause of cancer death among females [1].

Along with the improvement of living standards, people gradually pay more attentions to 
health problems. Spirulina platensis, known as "the 21st century's health products", has increasingly draw attention of more and more people [3]. With the rise of pharmacological research of natural products, marine extracts have increasingly become an important source of novel anti-tumor medicine. Many studies have confirmed that marine natural products have high-effective anti-cancer pharmacological effect, and have low-toxic or no toxic side effects [4]. C-phycocyanin (C-PC), a marine natural extract from Spirulina platensis, has been reported to have the pharmacological effects, such as anti-tumor, antioxidation, anti-inflammation and immune enhancing effects [5]. C-phycocyanin is easy to extract and purify, non-toxic and safe, and its pharmacological effects are extensive; therefore, it has a great research and development potential as a functional food or medicine. Whether it can be used as functional food or medicine, C-phycocyanin has important development and utilization in recent years [4]. Although more researches have been focused on the various pharmacological effects and mechanisms of C-phycocyanin, but the reports associated with the application of C-phycocyanin is not extensive and in-depth enough, and its poor stability put some limitations to the application in medicine. Therefore, the design of a safe, stable and effective C-phycocyanin delivery system is a top priority to solve the current problems.

The novel nano-drug opened up a new hot spot for medicine research, and nano-drug may be a new strategy for cancer treatment. Nano-drugs have the potential to overcome the shortcomings of conventional drugs in vivo [6]. The combination of C-phycocyanin and nanotechnology makes it possible to transfer the easily degradable protein. Carboxymethyl chitosan (CMC), formed by carboxylation modification of chitosan, has the characteristics of good water solubility, biodegradability, biocompatibility and non-toxic [7]. Thus CMC can serve as a nanomaterial to construct nano-drug. The combination of natural medicine or chemotherapy drugs and CMC has the advantages of high-efficacy, slow-release and low side effects [8]. We constructed the C-PC/CMC nanoparticles using the ionic crosslinking method, in which carboxymethyl chitosan acted as a carrier to package C-phycocyanin. The C-PC/CMC nanoparticles not only improved the C-phycocyanin stability, but also accomplished the slow release of C-phycocyanin, which would widen the application for the C-PC as an anti-cancer drug.

Tumor targeted drug delivery system has attracted more and more attention in the cancer treatment [9]. Tumor-targeted nano-drugs could specifically deliver nano-drugs to tumors and achieve the purpose of targeted administration. Thus, tumor-targeted nano-drugs could selective kill cancer cells. At present, tumor targeting nano-drugs have become a hot topic for cancer treatment. Some molecules which are specifically expressed and overexpressed in cancer cells, such as CD40, EGFR, HER2, CD44, ALDH, etc. [10-14], are considered to be a useful cancer molecular marker, which served as targeted molecules to construct tumor-targeted nano-drugs. Many reports have indicated that CD59 was highly expressed in many solid tumors, such as colon cancer [15], lung cancer [16], pancreatic cancer [17], and ovarian cancer [18], while CD59 was lowly expressed in normal cells [16]. CD59 is a kind of membrane complement regulatory protein, and play a role in suppressing the formation of complement attack complex (MAC), then further promote the immune escape of tumor cells [19]. Bing Li screened out CD59 specific ligand peptide (CD59sp) by phage peptide library, which could selectivity combine CD59 molecules [20]. Thus we constructed the C-PC/CMC-CD59sp nanoparticles in which CD59 acted as a targeted molecule.

A novel hepatocyte-targeted delivery system (CCS-NPs-GL) with glycyrrhizin (GL) surface modification based on N-caproyl chitosan (CCS) has been developed. The uptake of CCS-NPs-GL by rat hepatocytes was 2.1 times higher than that of non-GL-modified CCS-NPs in vivo. Furthermore, the content of adriamycin-loaded CCS-NPs-GL in the liver was 1.6 times higher than that of nonGLmodified CCS-NPs, which suggested that CCS-NPsGL was a stable and effective drug delivery vehicle for hepatocyte targeting [21]. In addition, the use of collagen peptide $(\mathrm{CP})$ for the preparation of chitosan $(\mathrm{CN}) \mathrm{NPs}$ is especially interesting, because $\mathrm{CP}$ has the function of stabilizing NPs under physiological conditions. Mono-dispersed $\mathrm{pH}$ responsive $\mathrm{CP}-\mathrm{CN}$ NPs of about $100 \mathrm{~nm}$ were prepared via ionic gelation method by simple and mild co-precipitation of $\mathrm{CP}$ and $\mathrm{CN}$. The $\mathrm{CP}-\mathrm{CN}-\mathrm{NPs}$ showed high encapsulation efficiency towards doxorubicin hydrochloride (DOX) as well as pH controlled release. Such NPs based on chitosan have high potential for use as smart drug delivery carriers in advanced cancer therapy [22].

Our laboratory took advantage of the technology of nanomedicine, and C-PC, CMC and CD59sp has successfully constructed the C-PC/CMC and C-PC/ CMC-CD59sp nanoparticles. CMC as a carrier, C-PC as an anti-tumor drug, CD59sp as a targeted molecule, the targeted C-PC/CMC-CD59sp NPs were constructed and targetedly delivered. Our pre-experiments had proved that the anti-tumor effect of C-PC/ CMC-CD59sp nanoparticles was better than C-PC and C-PC/CMC nanoparticles. This study further proved 
the effects of C-PC/CMC-CD59sp nanoparticles on cervical cancer in vitro and in vivo.

\section{Materials and Methods}

\section{Materials}

HeLa, SiHa, MDA-MB-231 and L929 cells were provided by the Affiliated Hospital of Qingdao University. C-phycocyanin was purchased from Taizhou Binmei Biotechnology Co., Ltd., Taozhou, China. Carboxymethyl chitosan (CMC) was purchased from Qingdao Honghai Bio-Tech Co. Ltd., Qingdao, China. CD59-specific ligand peptide was synthesized by Chinese Peptide Company, Hangzhou, China. CCK8 was purchased from Beijing Solarbio Science \& Technology, Beijing, China. Immunofluorescence staining kit was purchased from Boster Biological Engineering Co., Ltd. Rabbit antihuman cyclinD1, Bcl-2, caspase-3 and cleaved caspase-3 monoclonal antibodies were purchased from Abcam Company. All other chemicals reached analytical grade.

\section{Synthesis of C-PC/CMC NPs and C-PC/CMC- CD59sp NPs}

The C-PC/CMC NPs were synthesized by the ionic cross-linking method and $\mathrm{CaCl} 2$ acted as crosslinking agent. Firstly, $\mathrm{CaCl}_{2}$ was added dropwise into the mixed solution of $\mathrm{CMC}$ and C-PC until the solution became turbid, in which the ratio of $\mathrm{CMC}$ to C-PC $(w / w)$ was 3:1, the concentration of CMC was $2 \mathrm{mg} / \mathrm{mL}$, and the concentration of $\mathrm{CaCl}_{2}$ was $1 \mathrm{mg} / \mathrm{mL}$. Then, the mixed solution of CMC, C-PC and $\mathrm{CaCl}_{2}$ was constantly stirred at $4^{\circ} \mathrm{C}$, in the dark, for $30 \mathrm{~min}$. The synthesized C-PC/CMC NPs were separated by centrifugation at $12000 \times \mathrm{g}$ for $20 \mathrm{~min}$ at $4^{\circ} \mathrm{C}$. And the C-PC/CMC NPs were harvested by freeze-drying method at $-60^{\circ} \mathrm{C}$ [23].

The C-PC/CMC NPs were coupled with CD59sp by amine coupling, using EDC-activated carboxyl group conjugation. Firstly, 2mg EDC was added dropwise into1mg/mL C-PC/CMC NPs solution. The $\mathrm{pH}$ of the mixed solution was adjusted to 5.6, and then the mixed solution was constantly stirred at room temperature, in the dark for $1 \mathrm{~h}$. Then, $1 \mathrm{mg} / \mathrm{mL}$ CD59sp was added dropwise into the mixed solution of C-PC/CMC NPs. CD59sp and EDC was stirred overnight at $4^{\circ} \mathrm{C}$ in the dark. The C-PC/CMCCD59spNPs were subsequently separated by centrifugation at $12000 \times \mathrm{g}$ for $20 \mathrm{~min}$ at $4^{\circ} \mathrm{C}$. And the C-PC/CMC-CD59sp NPs were harvested by freeze-drying method at $-60^{\circ} \mathrm{C}[23]$.

\section{The experimental groups}

The experiment was divided into four groups: (1) control group, not treated with any drug; (2) C-PC treated group; (3) C-PC/CMCNPs treated group; and
(4) C-PC/CMC-CD59sp NPs treated group. Experimental drug concentration was $1 / 2$ of the IC50 value, and the ratio of drugs to cultures $(\mathrm{v} / \mathrm{v})$ was 1:1.

\section{CCK8 assay}

The effects of C-PC, C-PC/CMC NPs and C-PC/CMC-CD59sp NPs on HeLa, SiHa, MDA-MB231 and L929 cells were monitored using CCK8 assays. HeLa, SiHa, MDA-MB-231 and L929 cells $\left(1 \times 10^{4}\right.$ cells per well) were plated into 96-well cell culture plates overnight. Then the medium was replaced with fresh medium with various concentrations of drugs $(10,50,100,200 \mu \mathrm{g} / \mathrm{ml})$ for 24h. After treatment, the absorbance was detected at $490 \mathrm{~nm}$ by using the CCK8 following the manufacturer's instructions for $2 \mathrm{~h}$. The absorbance was positively correlated with cell viability. The IC50 values of drugs were calculated.

\section{Analysis of cell cycle by flow cytometry}

The HeLa and SiHa cells treated with C-PC, C-PC/CMC NPs and C-PC/CMC-CD59sp NPs were collected using $0.25 \%$ Trypsin, centrifuged at $800 \mathrm{rpm}$, and washed twice with cold PBS. The cells were resuspended in precooling $70 \%$ ethanol at $4^{\circ} \mathrm{Cfor} 4 \mathrm{~h}$. The fixed cells were incubated with propidium iodide solution $(20 \mu \mathrm{g} / \mathrm{mL}$ PI, $0.1 \%$ Triton X-100 staining solution, $0.1 \mathrm{mg} / \mathrm{mL}$ RNase A) for $30 \mathrm{~min}$. The cell cycle distribution was detected by the BD Biosciences FACSCanto II Analyzer. The number of cells per sample was at least $2 \times 10^{4}$. All experiments were repeated three or more times.

\section{Analysis of cell apoptosis by flow cytometry}

The HeLa and SiHa cells treated with C-PC, C-PC/CMCNPs and C-PC/CMC-CD59spNPs were collected and resuspended with $1 \times$ binding buffer at a density of $1 \times 10^{6}$ cells $/ \mathrm{mL}$. Equal volumes of Annexin V-FITC and propidium iodide (PI) were added and then incubated for $30 \mathrm{~min}$ at room temperature in the dark. The cell apoptosis was determined by the BD Biosciences FACS Canto II Analyzer.

\section{Analysis of Cellular uptake by flow cytometry}

The HeLa and SiHa cells treated with C-PC, C-PC/CMC NPs and C-PC/CMC-CD59sp NPs were collected, washed with cold PBS, fixed with $4 \%$ formaldehyde and washed with cold PBS again, finally resuspended to a density of $1 \times 10^{6}$ cells $/ \mathrm{mL}$. The cellular uptake was determined by the BD Biosciences FACS Canto II Analyzer.

\section{In vivo study: rat model bearing tumor}

The study was approved by institutional animal care committee of Qingdao University. The study was carried out in accordance with approved guidelines. 
A total of 20 adult BALB/c nude mice (females, 19-23g) were used for the experiment. SiHa cells ( $5 \times 10^{6}$ cells mixed with Basement Membrane Matrix) were subcutaneously injection into the axillary fossa of the nude mice. Tumor growth was measured daily with calipers. Tumor volume was calculated as $(\mathrm{L} \times \mathrm{W} 2) / 2$, where $\mathrm{L}$ is the length in millimeters, and $\mathrm{W}$ is the width in millimeters. When the tumors reached a mean volume of 80 to $110 \mathrm{~mm}^{3}$, the mice were randomly divided into four groups: control group, the C-PC/CMC NPs treated group and the C-PC/CMC-CD59spNPs treated group. These drugs are injected into the tumor area and tumor size was measured once every other day in a twenty-day experiment. At the end of treatment, all mice were executed and tumors were picked out and weighed. Animals were housed under standard conditions with ad libitum food and water with a 12:12 light: dark cycle at the Qingdao University facilities.

\section{Immunofluorescence analysis}

The slices were conventionally dewaxed, immersed in $0.01 \mathrm{M}$ citrate solution, then heated to boiling status in a microwave oven, and then permeabilized with $0.5 \%$ Triton X-100 for $15 \mathrm{~min}$. After washing with PBS for three times, the slide was blocked with $10 \%$ goat serum for $1 \mathrm{~h}$. Incubation with primary antibodies (1:200) was done overnight at $4{ }^{\circ} \mathrm{C}$. After washing, cells were exposed to the goat anti-rabbit secondary immunoglobulin for $1 \mathrm{~h}$ at room temperature. After being washed with PBS for three times, the cells were stained with SABC-Cy3 at a 1:100 dilution for 30min in dark. After washing, the nuclei were stained with DAPI for $7 \mathrm{~min}$ before imaging. The slides were observed under a fluorescence microscope.

\section{Western blot}

The concentrations of protein in the samples were quantified using a BCA protein assay kit (Beyotime Biotechnology, Shanghai, China). First, $40 \mu \mathrm{g}$ protein was mixed with SDS loading buffer $(6 \times)$ and then boiled for $10 \mathrm{~min}$. Then the obtained protein samples were separated by 10\% SDS-PAGE, transferred to PVDF membrane (Millipore), and blocked with $5 \%$ defatted dry milk in TBS-Tween $20(0.1 \%, \mathrm{v} / \mathrm{v})$ for 1 hour at room temperature and then incubated with specific primary antibodies at $4^{\circ} \mathrm{C}$ for more than $16 \mathrm{~h}$. After washing five times with Tris-buffered saline containing 1\% (v/v) Tween-20 (TBST), the membrane was incubated with the appropriate horseradish peroxidase secondary antibody for 1 hour. Following several washes, the blots were detected by an enhanced chemiluminescence (Millipore).

\section{Terminal dUTP nick-end labeling (TUNEL) assay}

For the detection of DNA integrity, HeLa and SiHa cells were stained with TUNEL detection kit. The collected cells were washed with PBS, and then fixed with $4 \%$ paraformaldehyde / PBS solution (pH7.4) at room temperature for $15 \mathrm{~min}$. After fixation, the collected cells were immersed into $0.3 \%$ Triton X-100 PBS solution at room temperature for 15 min. The collected cells were incubated with $50 \mu 1$ labeling reaction mixture $(5 \mu \mathrm{TdT}$ Enzyme and $45 \mu 1$ TUNEL fluorescent labeling buffer) in a $37^{\circ} \mathrm{C}$ humidified chamber for $60 \mathrm{~min}$, and then washed twice with PBS. After labeling, HeLa and SiHa cells were counterstained with DAPI and visualized under a fluorescence microscopy.

\section{Statistics analysis}

The experiments were performed three or four times independently. Results were represented as mean \pm standard deviation (SD). Statistical analyses were carried out by the Student's t test or the one-way analysis of variance (ANOVA) using a statistical software package (SPSS, USA). $\mathrm{P}<0.05$ was considered as statistically significant. Statistical significance was also taken as* $\mathrm{P}<0.05$ and ${ }^{* *} \mathrm{P}<0.01$.

\section{Results}

\section{Nanoparticles further inhibited the proliferation of cervical cancer cells}

The cytotoxic effects of C-PC, C-PC/CMC NPs and C-PC/CMC-CD59sp NPs on cervical cancer HeLa and SiHa cells, breast cancer MDA-MB-231 cells and mouse fibroblast cell line L929 were firstly evaluated by the CCK8 cell viability assay. The cell viabilities were reduced in a dose-dependent manner in HeLa, SiHa, MDA-MB-231 and L929 cell lines after the treatment of C-PC, C-PC/CMC NPs and C-PC/CMCCD59sp NPs for 24h. As shown in Figure 1A, the IC50 values of C-PC, C-PC/CMC NPs, and C-PC/CMCCD59sp NPs for HeLa cells were 1104.0, 116.5, $50.64 \mu \mathrm{g} / \mathrm{ml}$, respectively. Similarly, as shown in Figure 1B, the IC50 values of C-PC, C-PC/CMC NPs, C-PC/CMC-CD59sp NPs for SiHa cells were 375.8, $79.67,20.37 \mu \mathrm{g} / \mathrm{ml}$. And as shown in Figure $1 \mathrm{C}$, the IC50 values of C-PC, C-PC/CMC NPs, and C-PC/CMC-CD59sp NPs for MDA-MB-231 cells were 294.0, 115.8, $47.29 \mu \mathrm{g} / \mathrm{ml}$, respectively. Similarly, as shown in Figure 1D, the IC50 values of C-PC, C-PC/CMC NPs, C-PC/CMC-CD59sp NPs for L929 cells were $596.0,458.2,259.60 \mu \mathrm{g} / \mathrm{ml}$. The inhibition effect of C-PC/CMC-CD59sp NPs was significantly higher than the C-PC and C-PC/CMC NPs. Compared with HeLa, MDA-MB-231 and L929 cells, 
the nanoparticles showed higher toxic to SiHa cells, especially C-PC/CMC-CD59sp NPs. In view of the above results, our data suggested that the antiproliferation effects of C-PC packaged in nanoparticles were well reserved and further enhanced in targeted nanoparticles.

\section{Nanoparticles further induced G0/G 1 cell cycle arrest of cervical cancer cells}

Since C-PC, C-PC/CMC NPs and C-PC/CMCCD59sp NPs inhibited cervical cancer HeLa and SiHa cells proliferation, we further explored the effects of nanoparticles on cell cycle progress. The synchronized cells were treated with C-PC, C-PC/CMC NPs and C-PC/CMC-CD59sp NPs for $24 \mathrm{~h}$, and then followed by flow cytometry analysis to examine the cell DNA contents. As shown in Figure 2A, the G1 peaks of HeLa cells increased from $41.81 \%$ (control), $48.98 \%$ (C-PC), 54.04\% (C-PC/CMC NPs) to $63.29 \%$ (C-PC/
CMC-CD59spNPs) after the treatment, respectively. In addition, the accumulation of cells in the G1 phase was accompanied by a decrease in the population of HeLa cells in the $S$ and G2phase. As shown in Figure 2B, the G1 peaks of SiHa cells increased from $42.63 \%$, $48.26 \%, 55.69 \%$ to $65.59 \%$ after treated with PBS, C-PC, C-PC/CMC NPs and C-PC/CMC-CD59sp NPs, respectively. Similarly, the accumulation of $\mathrm{SiHa}$ cells in the G1 phase was accompanied by a decrease in the population of cells in the S and G2 phase. Therefore, these results indicated that the C-PC/CMC-CD59sp NPs significantly induced G0/G1 cell cycle arrest of cervical cancer HeLa and SiHa cells compared with C-PC and C-PC/CMC NPs. Moreover, compared with HeLa cells, the effects of G0/G1 cell cycle arrest induced by nanoparticles, especially C-PC/CMCCD59sp NPs, were more significant for SiHa cells.
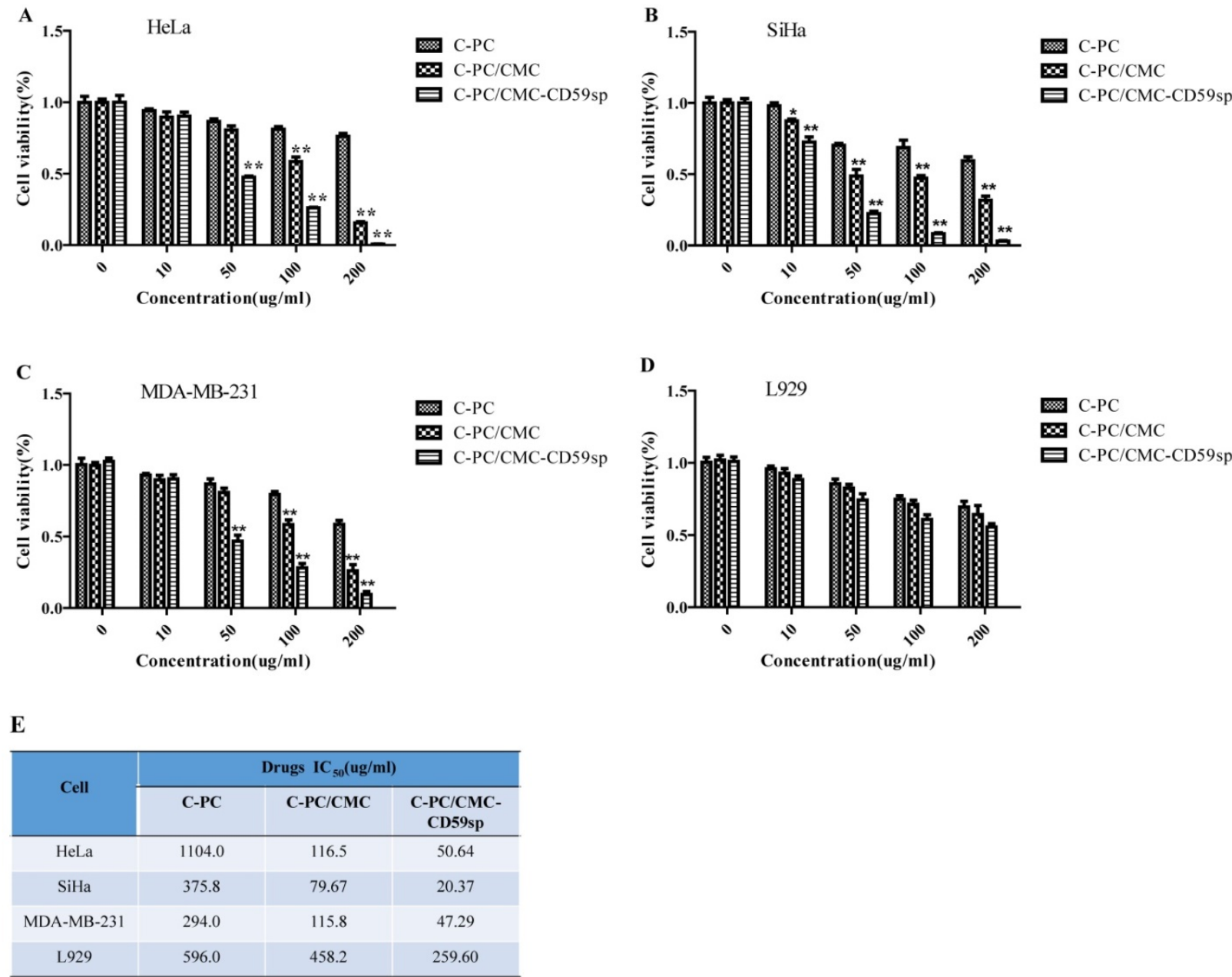

Figure 1. Growth inhibition effect of nanoparticles in HeLa, SiHa, MDA-MB-231 and L929 cells. (A) Cell viability of HeLa cells is detected by CCK8 assay after treatment with C-PC, C-PC/CMC NPs and C-PC/CMC-CD59sp NPs for 24h. (B) Cell viability of SiHa cells is detected by CCK8 assay. (C) Cell viability of MDA-MB-231 cells is detected by CCK8 assay. (D) Cell viability of L929 cells is detected by CCK8 assay. Six samples were analyzed in each group, and the results are presented as the mean \pm SD. (E) IC50 values of C-PC, C-PC/CMC NPs and C-PC/CMC-CD59sp NPs in HeLa, SiHa, MDA-MB-231 and L929 cells after treatment for $24 \mathrm{~h}$. 


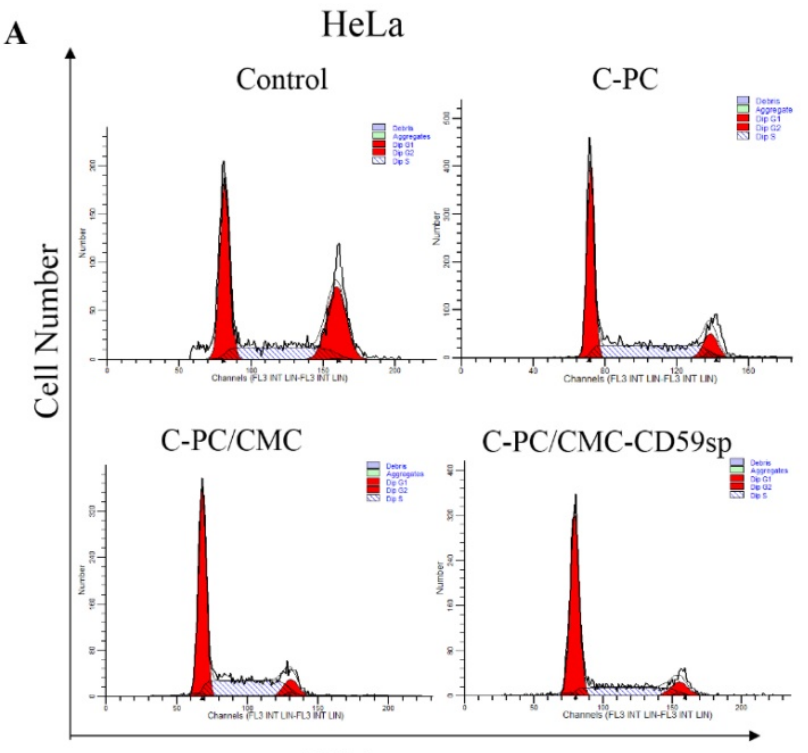

DNA content

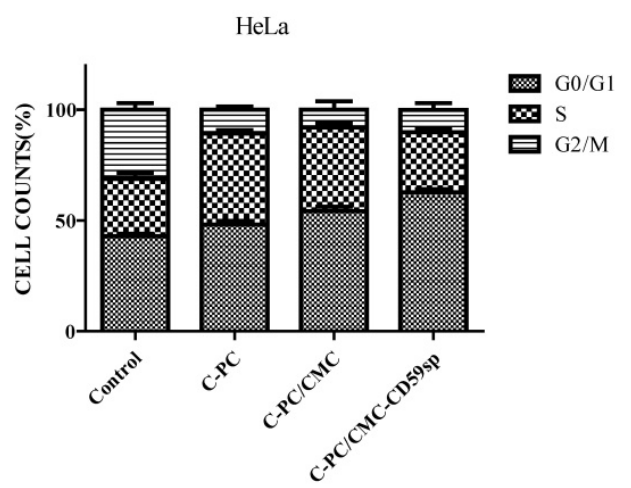

B $\quad \mathrm{SiHa}$

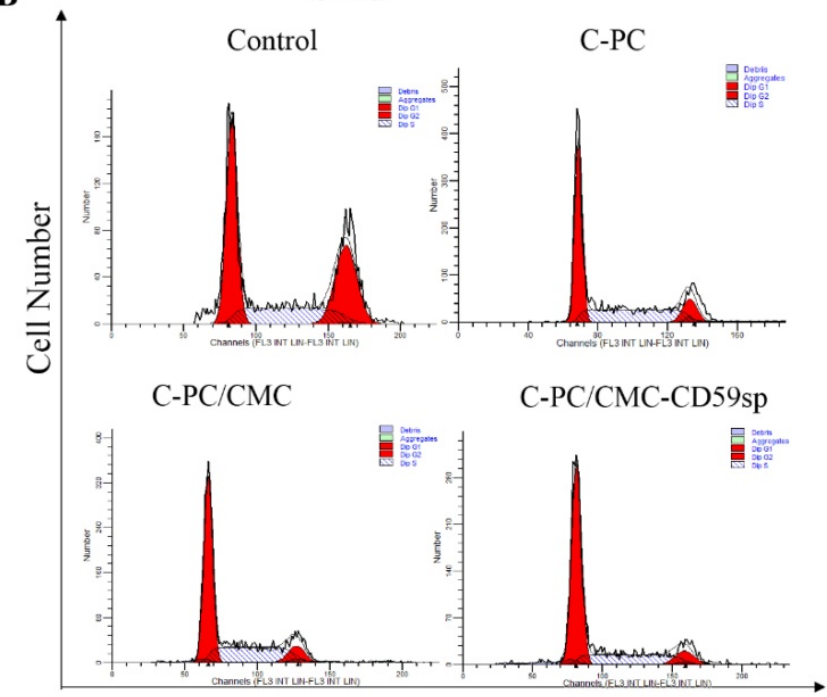

DNA content

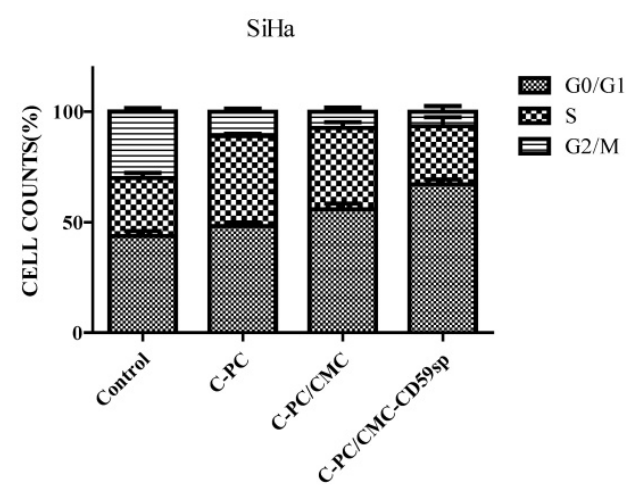

Figure 2. Effects of nanoparticles on cell cycle distribution in HeLa and SiHa cells. (A) C-PC, C-PC/CMC NPs and C-PC/CMC-CD59sp NPs induced G0/Gl cell cycle arrest in HeLa cells. Quantitative representation of cell cycle distribution after treatment for 24h. (B) C-PC, C-PC/CMC NPs and C-PC/CMC-CD59sp NPs induced G0/GI cell cycle arrest in SiHa cells. Quantitative representation of cell cycle distribution after treatment for $24 \mathrm{~h}$.

\section{Nanoparticles further induced cellular apoptosis of cervical cancer cells}

To evaluate whether C-PC, C-PC/CMC NPs and C-PC/CMC-CD59sp NPs could induce apoptosis in cervical cancer, HeLa and $\mathrm{SiHa}$ cells were stained with Annexin V-FITC/PI, and then detected by flow cytometry. As shown in Figure 3A, the percentage of early apoptotic cells increased gradually from $6.3 \%$ (control) to $6.4 \%$ (C-PC), $12.9 \%$ (C-PC/CMC NPs) and $15.5 \%$ (C-PC/CMC-CD59spNPs) in HeLa cells after the treatment of nanoparticles for 24 hours. Consistent with this result, the percentage of early apoptotic cells increased gradually from $10.0 \%$ (control) to $10.4 \%$ (C-PC), $14.5 \% \quad$ (C-PC/CMC NPs) and $18.1 \%$ (C-PC/CMC-CD59spNPs) in SiHa cells after the treatment of nanoparticles for 24 hours (Figure 3B). So the nanoparticles could induce early apoptosis (Annexin V+/PI-) in HeLa and SiHa cells.

In order to further study the pro-apoptotic effect of C-PC, C-PC/CMC NPs and C-PC/CMC-CD59sp
NPs on cervical cancer HeLa and SiHa cells, TUNEL assay was carried out. The TUNEL assay showed that the TUNEL-positive cells were significant increased when HeLa and SiHa cells were treated with C-PC/CMC NPs and C-PC/CMC-CD59sp NPs (Figure 3C and 3D). Compared with C-PC and C-PC/ CMC NPs, the pro-apoptosis effect of C-PC/CMCCD59sp NPs was more significant for cervical cancer HeLa and SiHa cells. In view of the above results, our data suggested that the pro-apoptosis effects of C-PC packaged in nanoparticles were more significant, and further enhanced in targeted nanoparticles C-PC/ CMC-CD59sp NPs.

\section{C-PC/CMC-CD59sp NPs specifically combined with cervical cancer HeLa and SiHa cells}

The targeted nanoparticles C-PC/CMC-CD59sp NPs were constructed with C-PC, CMC and CD59sp, and under the guidance of CD59sp, C-PC/CMCCD59sp NPs could specifically combine with CD59 on 
HeLa and SiHa cells surface. Afterwards, C-PC/ CMC-CD59sp NPs accumulated on the HeLa and $\mathrm{SiHa}$ cells surface, and then were engulfed into cells and exerted anti-cancer functions. The intakes of nanoparticles into HeLa and SiHa cells were observed after treated with C-PC, C-PC/CMC NPs and C-PC/CMC-CD59sp NPs. C-PC emits red fluorescence itself, and with the increase of C-PC concentration, red fluorescence intensity was also increased. As shown in Figure 4A, the fluorescence intensity of HeLa cells treated with C-PC/CMC-
CD59sp NPs was significantly more than control group, C-PC treated group and C-PC/CMC NPs treat group. Similarly, we also found that the fluorescence intensity of SiHa cells treated with C-PC/CMCCD59sp NPs was significantly more than control group, C-PC treated group and C-PC/CMC NPs treat group (Figure 4B). Thus, those results suggested the CD59sp could promote C-PC/CMC-CD59sp NPs specifically to combine with CD59 on HeLa and SiHa cells.

\begin{tabular}{c|c|c|c|c|}
\hline \multirow{2}{*}{ Cell } & \multicolumn{5}{|c}{$(\%)$ OF EARLY APOPTOSIS CELLS } \\
\cline { 2 - 5 } & Control & C-PC & C-PC/CMC & $\begin{array}{c}\text { C-PC/CMC- } \\
\text { CD59sp }\end{array}$ \\
\hline HeLa & 6.3 & 6.4 & 12.9 & 15.5 \\
\hline SiHa & 10.0 & 10.4 & 14.5 & 18.1 \\
\hline
\end{tabular}

A

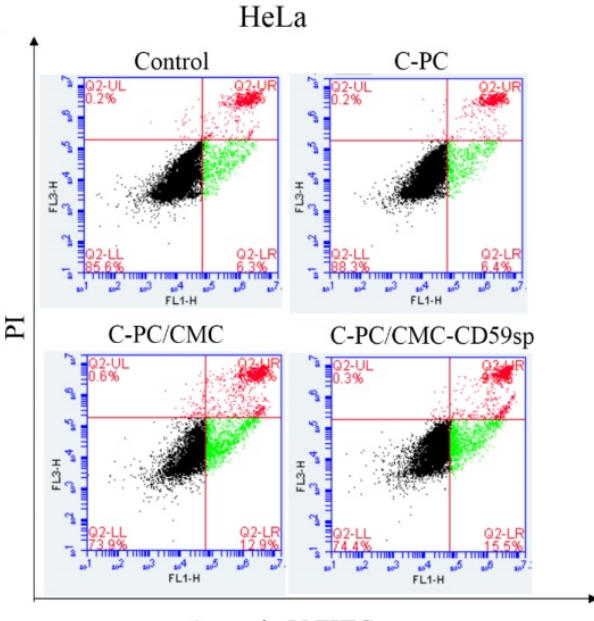

Annexin V-FITC$$
\text { c }
$$
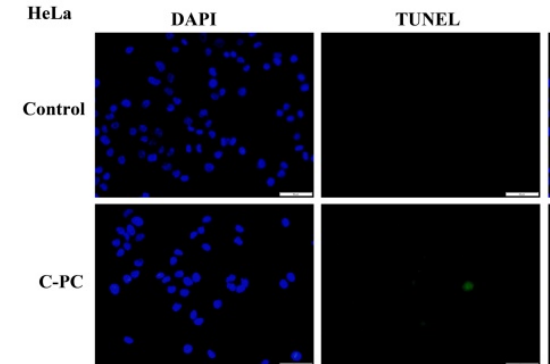

C-PC/CMC

tons
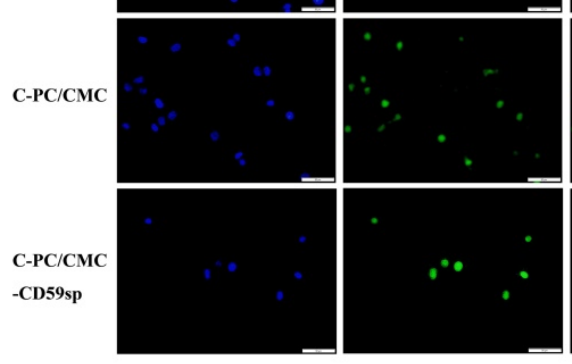

B

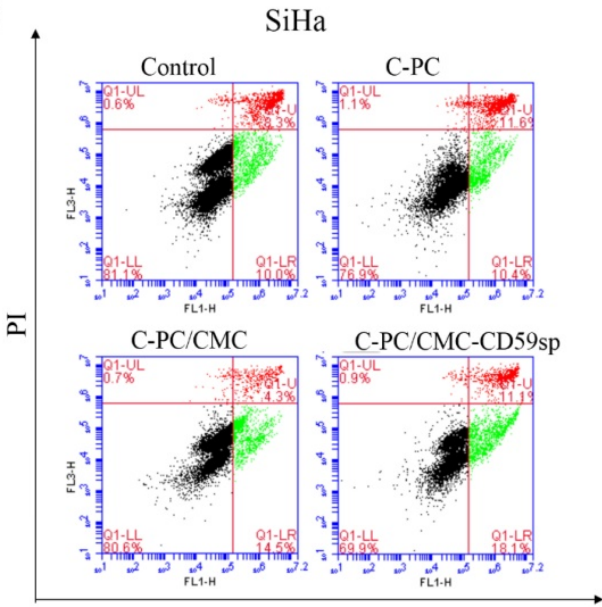

Annexin V-FITC

D

MERGE

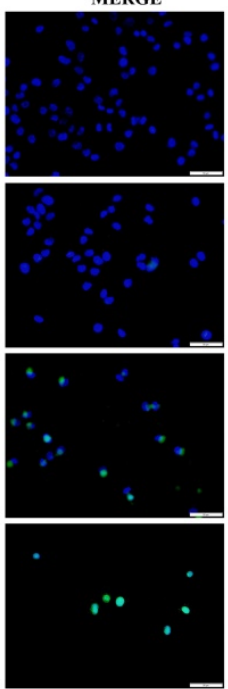

SiHa

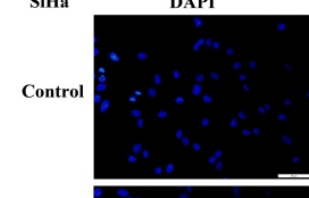

C-PC

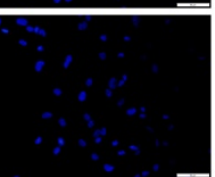

C-PC/CMC
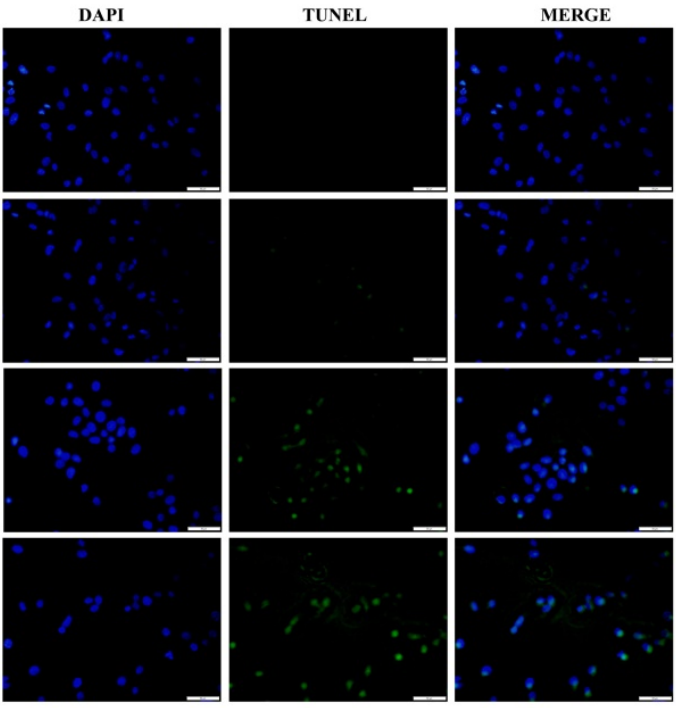

Figure 3. Effect of nanoparticles on cell apoptosis in HeLa and SiHa cells. (A)Analysis of HeLa cells apoptosis by flow cytometry using Annexin V-FITC and Pl after treatment with C-PC, C-PC/CMC NPs and C-PC/CMC-CD59sp NPs for 24h. (B)Analysis of SiHa cells apoptosis by flow cytometry using Annexin V-FITC and PI. Quantitative representation of early apoptotic cells after treatment for 24h. (C) Analysis of HeLa cells apoptosis by TUNEL assay after treatment with C-PC, C-PC/CMC NPs and C-PC/CMC-CD59sp NPs for 24h. (D) Analysis of HeLa cells apoptosis by TUNEL assay. Green fluorescence represents TUNEL-positive cells after treatment. 
A

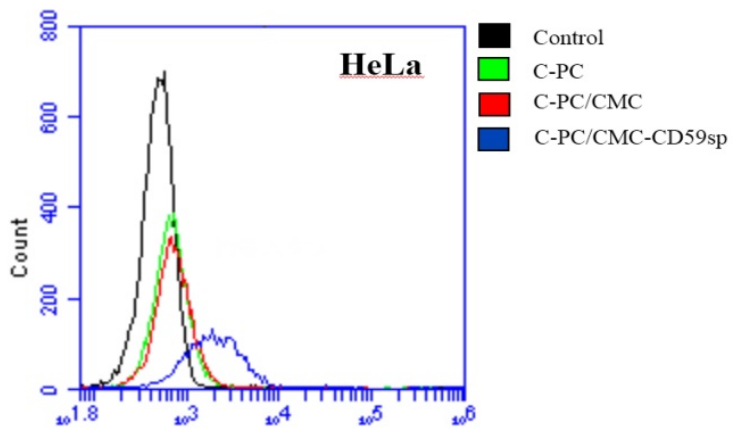

B

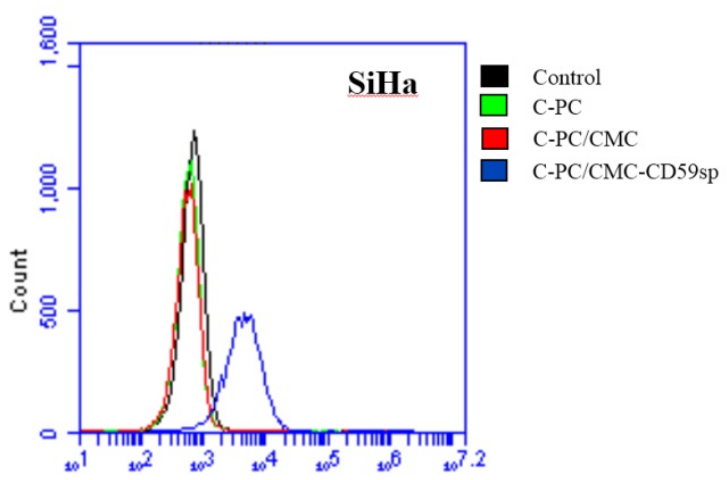

Figure 4. Targeting effect of nanoparticles in $\mathrm{HeLa}$ and $\mathrm{SiHa}$ cells. (A) The intakes of nanoparticles were detected by flow cytometry in HeLa cells after treated with C-PC, C-PC/CMC NPs and C-PC/CMC-CD59sp NPs. (B) The intakes of nanoparticles were detected by flow cytometry in $\mathrm{SiHa}$ cells.

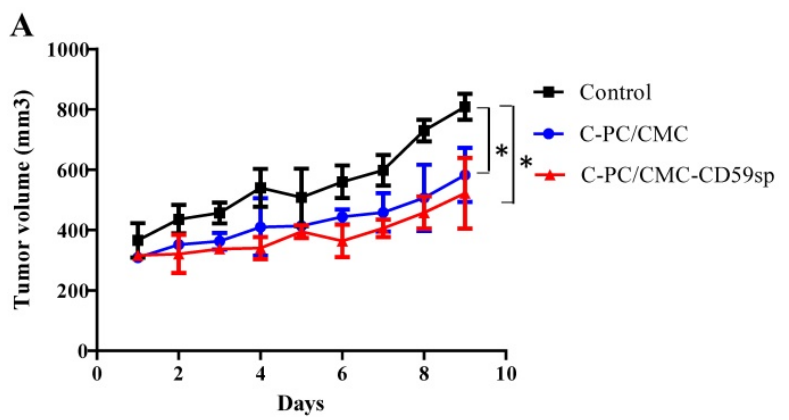

B

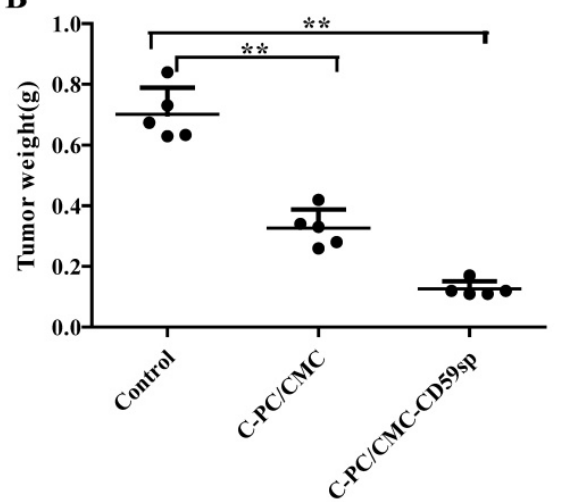

Figure 5. Nanoparticles suppressed cervical cancer tumor growth in vivo. (A) Tumor volumes of xenograft tumors from mice treated with C-PC/CMC NPs and C-PC/CMC-CD59sp NPs. (B) Tumor weights of xenograft tumors from mice treated with C-PC/CMC NPs and C-PC/CMC-CD59sp NPs. Data were presented as the mean $\pm S D, n=5$ for each group.

\section{Nanoparticles suppressed cervical cancer tumor growth in vivo}

Subsequently, we evaluated the antitumor effect of C-PC/CMC NPs and C-PC/CMC-CD59sp NPs in vivo on SiHa cells based tumor xenograft model. Nude mice bearing subcutaneous $\mathrm{SiHa}$ cells tumor xenograft were treated with PBS (control), CMC/C-PCNPs and CMC/C-PC-CD59sp NPs ( $n=5)$. Compared to the controls, none of the mice in the C-PC/CMC NPs and CMC/C-PC-CD59sp NPs treat group exhibited overt toxicity and any body weight loss. In Figure 5A, the growth of $\mathrm{SiHa}$ tumor xenografts was progressively suppressed in the CMC/C-PC NPs and CMC/C-PCCD59sp NPs treatment groups. Moreover, the inhibitory effects of CMC/C-PC-CD59sp NPs were more significant than CMC/C-PC NPs. We found that the weights of $\mathrm{SiHa}$ tumor xenografts were significantly reduced in the CMC/C-PC NPs and CMC/C-PCCD59sp NPs treatment groups. And the tumors weights in the CMC/C-PC-CD59sp NPs treatment groups were lighter than CMC/C-PC NPs (Figure 5B). These results suggested that the C-PC/CMC NPs and CMC/C-PC-CD59sp NPs could suppress cervical cancer tumor growth in vivo, and the inhibitory effects of C-PC/CMC-CD59sp NPs were more significant.

\section{Nanoparticles regulated Bcl-2/Cleaved caspase-3 protein expression of cervical cancer tumor xenograft in vivo}

The C-PC/CMC NPs and C-PC/CMC-CD59sp NPs could play anti-cancer roles in cervical cancer tumor xenograft in vivo, and cell apoptosis is one important mechanism of cell death, so we detected the protein expression of apoptosis-related proteins, such as Bcl-2 (an anti-apoptotic protein) and cleaved caspase-3 (an pro-apoptotic protein). As shown in Figure 6A, compared with the control group, the Bcl-2 protein expression levels of the CMC/C-PC NPs and CMC/C-PC-CD59sp NPs treatment groups were significantly reduced, and $\mathrm{Bcl}-2$ protein level of the CMC/C-PC-CD59sp NPs treatment groups was lower than CMC/C-PC NPs. In contrast to Bcl-2 protein, the cleaved caspase-3 protein expression levels of the CMC/C-PC NPs and CMC/C-PC-CD59sp NPs treatment groups were significantly increased, especially in CMC/C-PC-CD59sp NPs treatment groups (Figure 6B). Consistent with immunofluorescence results, compared with control group, cleaved caspase-3 expression levels of the CMC/C-PC NPs and CMC/C-PC-CD59sp NPs treatment groups were significantly increased, while Bcl-2 protein expression levels were significantly reduced, and Bcl-2/cleaved caspase-3 in the C-PC/CMC-CD59sp NPs treatment group were more significant than CMC/C-PC NPs (Figure 6C).Taken together, these results suggested 
that the C-PC/CMC NPs and C-PC/CMC-CD59sp NPs could up-regulate cleaved caspase-3 protein expression and down-regulate $\mathrm{Bcl}-2$ protein expression, and C-PC/CMC-CD59sp NPs could further promote transduction of apoptotic signals.

\section{Nanoparticles regulated p21/CDK4/Cyclin D1 protein expression of cervical cancer tumor xenograft in vivo}

Since the C-PC/CMC NPs and C-PC/CMCCD59sp NPs induced G0/G1 phase cell cycle arrest in cervical cancer HeLa and SiHa cells, we further explored the protein levels of cell cycle regulatory proteins involved in G1 to $S$ transition which included Cyclin D1, CDK4 and p21 in cervical cancer tumor xenograft in vivo. Furthermore, CDKIs (CDK inhibitor p21) were often associated with the suppression of CDKs activity by forming CDK-CDKI complexes [24]. We found the protein expression levels of Cyclin D1 and CDK4 in the C-PC/CMC NPs and C-PC/CMCCD59sp NPs treatment group were significantly decreased, as shown in Figure 7, while the expression level of p21 in the C-PC/CMC NPs and C-PC/ CMC-CD59sp NPs treatment group were significantly increased. In addition, $\mathrm{p} 21 / \mathrm{CDK} 4 / \mathrm{Cyclin} \mathrm{D} 1$ protein expression level changes in the C-PC/CMC-CD59sp NPs treatment group were more significant than
CMC/C-PC NPs. All these results suggested C-PC/ CMC NPs and C-PC/CMC-CD59sp NPs could up-regulate p21 proteins expression, further inhibit the expressions of Cyclin D1 and CDK4 in cervical cancer tumor xenograft in vivo.

\section{Nanoparticles inhibited MMP-2 protein expression of cervical cancer tumor xenograft in vivo}

The MMP-2 protein was reported as an angiogenic and metastatic inducers in many cancers [25]. Hence, MMPs are considered as a therapeutic target in many diseases [26]. Since it was reported that C-PC selectively inhibited MMPs (MMP-2 and MMP-9) in HepG2 cells, we further explored the protein level of MMP-2 in cervical cancer tumor xenograft in vivo. We found the MMP-2 protein expression in the C-PC/CMC NPs and C-PC/ CMC-CD59sp NPs treatment group were significantly decreased, as shown in Figure 8, and MMP-2protein expression level change in the C-PC/CMC-CD59sp NPs treatment group was more significant than CMC/C-PC NPs. These results suggested C-PC/CMC NPs and C-PC/CMC-CD59sp NPs could downregulate MMP-2 protein expression in cervical cancer tumor xenograft in vivo, especially C-PC/CMCCD59sp NPs.
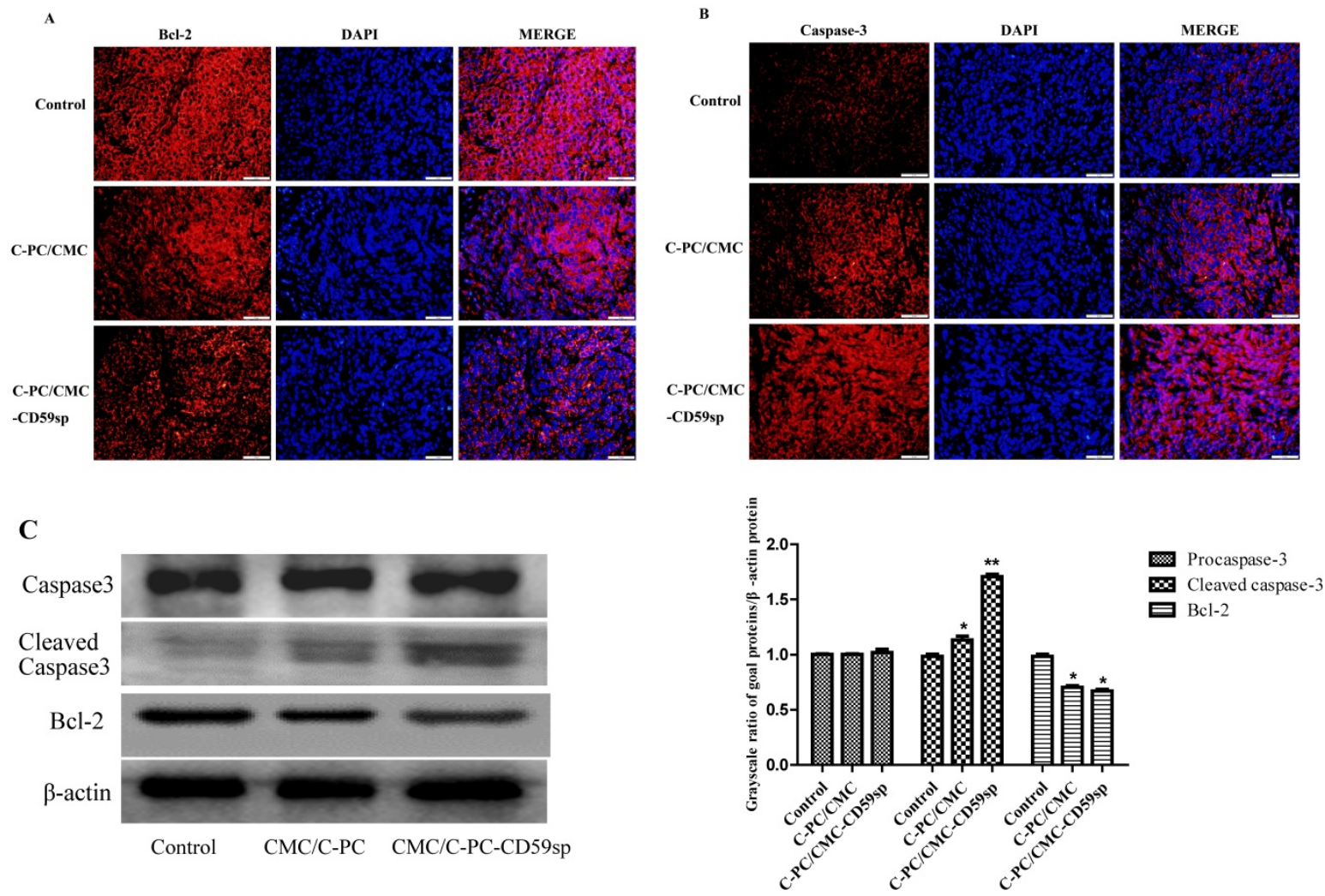

Figure 6. Nanoparticles regulated Bcl-2/ Cleaved caspase-3 protein level in vivo. (A) Analysis of Bcl-2 protein level in vivo by immunofluorescent assay. Nanoparticles down-regulated Bcl-2 protein level in vivo. (B) Analysis of Cleaved caspase-3 protein level in vivo by immunofluorescent assay. Nanoparticles up-regulated Cleaved caspase-3 protein level in vivo. (C) Analysis of Bcl-2/Cleaved caspase-3 protein level in vivo by Western blotting. Quantitative representation of grayscale ratio of target protein/ $\beta$-actin. $* \mathrm{P}<0.05$ vs control group and $* * \mathrm{P}<0.01$ vs control group. 
A

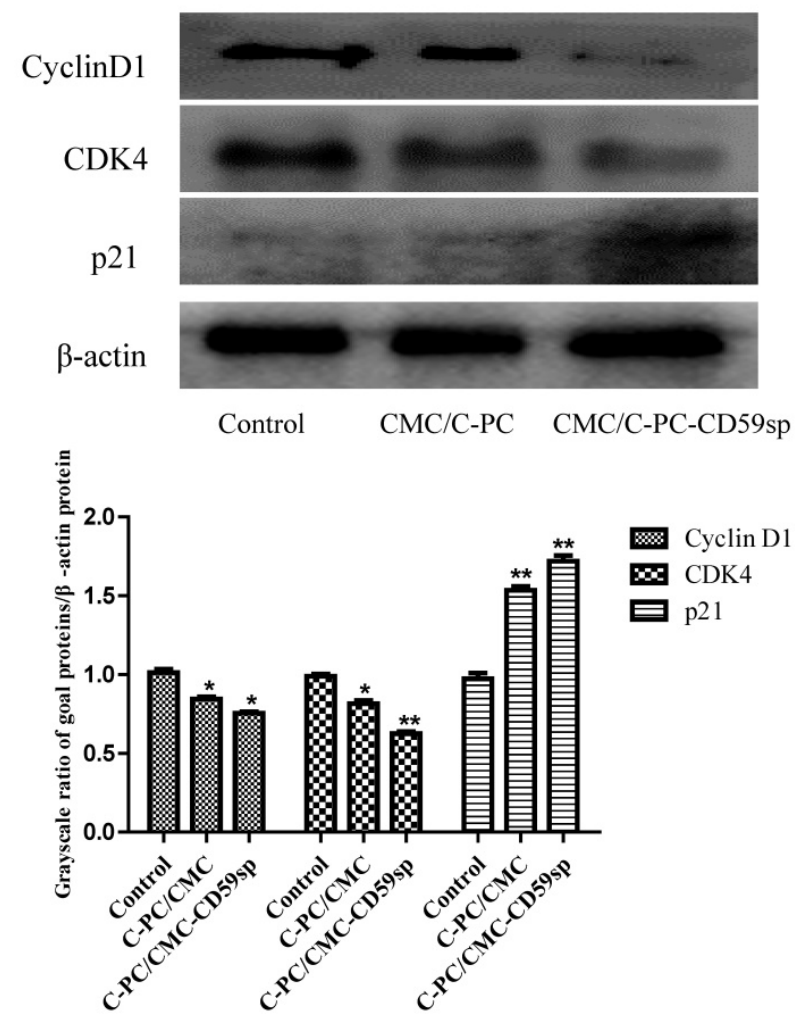

Figure 7. Nanoparticles regulated the expressions of Cyclin DI, CDK4, and p21. (A) Analysis of Cyclin D1, CDK4, and p21 protein levels in vivo by Western blotting. Quantitative representation of grayscale ratio of target protein $/ \beta$-actin. $* \mathrm{P}<0.05$ vs control group and $* * \mathrm{P}<0.01$ vs control group.

A
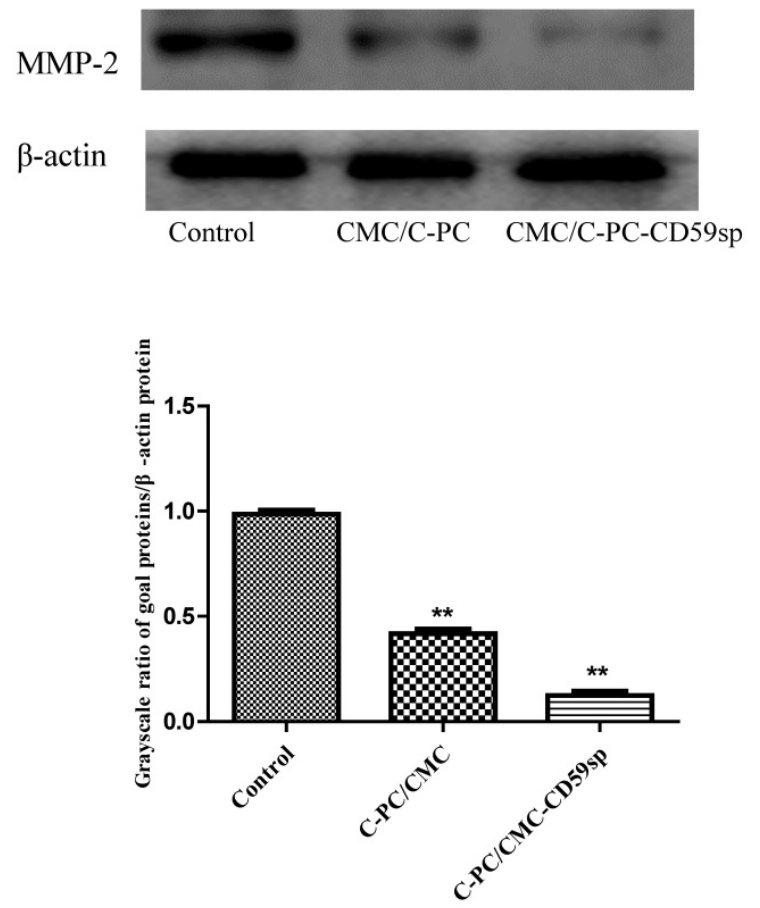

Figure 8. Nanoparticles regulated the expressions of MMP-2. (A) Analysis of MMP-2 protein level in vivo by Western blotting. Quantitative representation of grayscale ratio of target protein $/ \beta$-actin. $* P<0.05$ vs control group and $* * \mathrm{P}<0.01$ vs control group.

\section{Discussion}

Cervical cancer is one of the most common gynecological cancers and one of the leading causes of gynecological cancer lethality in women worldwide $[27,28]$. Radiotherapy and surgery can be used as the main treatment of cervical cancer. The standard therapy for patients with more advanced disease is external beam radiotherapy and concurrent chemotherapy, followed by brachy therapy [29]. Unfortunately, the standard therapy is not available in many undeveloped countries, so over $85 \%$ of cases and $90 \%$ of deaths occur in undeveloped countries [30]. Although patients with early cervical cancer can be treated with radiation and surgery, advanced cervical cancer is almost incurable, so new treatments for cervical cancer are needed. Currently, some anti-tumor medicines cannot be widely used in clinical therapy because of their short effective half-lives and serious side effects. In addition, conventional chemotherapeutic drugs often have many serious side effects during the treatment of cancer. Consequently, the search for new anti-cancer drugs with lower side effects and higher efficacy is urgently desirable. C-phycocyanin is an ideal anti-tumor agent we are looking for. C-phycocyanin, a water-soluble phycobiliprotein extracted from Spirulina platennis, is commonly used in cosmetic colorants, functional food and medicine. It has been proved that C-PC has important pharmacological functions, such as anti-oxidation, anti-inflammation, photo-induced cytotoxicity, antitumor, and immunological enhancement [4, 5]. It has been reported that C-PC has effective anti-cancer functions on various cancer cell types (such as breast cancer, bone marrow cancer, colon cancer, lung cancer, liver cancer, and Leukemia) [4]. These evidences confirmed the therapeutic potential of C-PC in cancer therapy. However, as a protein, C-PC has some shortcomings such as poor stability, easy degradation, sensitive to light and temperature shortcomings, which put some limitations to the further application of C-PC in medicine.

To solve the shortcomings of C-PC, our laboratory wanted to look for a safe, stable and effective C-PC delivery system, so we designed a nano-drug to packaged C-PC. Currently, the nano-drugs, the combination of biomedicine and nanotechnology, have become a new trend in drug development [6]. The nano-drugs may develop into a new strategy for cancer treatment that overcomes the shortcomings of traditional medicines. Thus, the combination of C-phycocyanin and nanotechnology makes it possible to take advantage of C-phycocyanin and overcome the disadvantages of C-phycocyanin. After long-term research, our laboratory took 
advantage of the technology of nanomedicine, and successfully constructed the C-PC/CMC and C-PC/CMC-CD59sp nanoparticles using the ionic crosslinking method for the first time, in which CMC acted as a carrier to package C-PC, C-PC acted as an anti-cancer drug, and CD59sp acted as a targeted molecule to specifically bind to CD59 [3, 23]. The C-PC/CMC and C-PC/CMC-CD59sp nanoparticles not only improved the stability and efficacy of C-PC, but also achieved the slow release of C-PC. Furthermore, in C-PC/CMC-CD59sp nanoparticles, CD59sp functioned as a targeted molecule to transport C-PC/CMC-CD59sp NPs to tumor to achieve the tumor-target. Peng Yang found the C-PC/CMC-CD59sp nanoparticles efficiently targeted the surface of HeLa cells and had a significant inhibitory effect on HeLa cell proliferation [23]. The C-PC/CMC-CD59sp nanoparticles induced HeLa cells apoptosis by up-regulation of cleaved caspase-3 and cleaved poly ADP-ribose polymerase proteins, and down-regulation of $\mathrm{Bcl}-2$ proteins in vitro [3]. Yujuan Wang found the C-PC/CMC and C-PC/ CMC-CD59sp nanoparticles were well distributed with a diameter distribution of $200 \pm 11.3 \mathrm{~nm}$ and zeta potential of $-19.5 \pm 4.12 \mathrm{mV}$. And the C-PC/CMC and C-PC/CMC-CD59sp nanoparticles could inhibit cervical cancer tumor growth in HeLa cells tumor xenograft in vivo [3]. The C-PC/CMC and C-PC/ CMC-CD59sp nanoparticles could induce cell apoptosis by up-regulation of cleaved caspase-3 proteins expression, and down-regulation of $\mathrm{Bcl}-2$ and cyclinD1 proteins in cervical cancer tumor xenograft in vivo [3]. Our laboratory provided a new idea for the research and development of C-PC, and supplied a new application for the C-PC as an anti-cancer drug.

A novel hepatocyte-targeted delivery system (CCS-NPs-GL) with glycyrrhizin (GL) surface modification based on N-caproyl chitosan (CCS) has been developed. N-caproyl chitosan (CCS) was synthesized by acylation of amino group of chitosan, and glycyrrhizin (GL) was oxidized to be conjugated to the surface of N-caproyl chitosan nanoparticles. The prepared CCS-NPs-GL nanoparticles exhibited an associated adriamycin quite efficiently (association efficiency: $87.5 \%$ ). The prepared CCS-NPs-GL nanoparticles also possessed dimensional and glycyrrhizin surface-binding stability and slow release property in plasma in vitro. The uptake of CCS-NPs-GL by rat hepatocytes was 2.1 times higher than that of non-GL-modified CCS-NPs in vivo. Furthermore, the content of adriamycin-loaded CCS-NPs-GL in the liver was 1.6 times higher than that of nonGL-modified CCS-NPs, which suggested that CCS-NPs-GL was a stable and effective drug delivery vehicle for hepatocyte targeting [21].

In addition, the use of collagen peptide (CP) for the preparation of chitosan $(\mathrm{CN})$ NPs is especially interesting, because $\mathrm{CP}$ has the function of stabilizing chitosan (CN) NPs under physiological conditions. Mono-dispersed $\mathrm{pH}$ responsive CP-CN-NPs of about $100 \mathrm{~nm}$ were prepared via ionic gelation method by simple and mild co-precipitation of $\mathrm{CP}$ and $\mathrm{CN}$. Hydrogen bonding and electrostatic interactions are believed to be major driving forces for NP formation and drug encapsulation, respectively. The $\mathrm{CP}-\mathrm{CN}-$ NPs showed high encapsulation efficiency towards doxorubicin hydrochloride (DOX) as well as $\mathrm{pH}$ controlled release. The study found that DOX loaded CP-CN-NPs showed excellent anti-proliferative characteristics against HeLa cells with favorable biocompatibility against normal cells. Such NPs based on chitosan have high potential for use as smart drug delivery carriers in advanced cancer therapy [22].

In our study, we found that compared with C-PC, the C-PC/CMC and C-PC/CMC-CD59sp NPs could significantly inhibit the proliferation, promote cell apoptosis and induce G0/G1 cell cycle arrest in cervical cancer HeLa and $\mathrm{SiHa}$ cells in vitro. The inhibition effect of C-PC/CMC-CD59sp NPs was significantly higher than the C-PC/CMC NPs. And the C-PC/CMC and C-PC/CMC-CD59sp NPs could induce G0/G1 cell cycle arrest in cervical cancer HeLa and SiHa cells, especially C-PC/CMC-CD59sp NPs. The pro-apoptosis effects of the C-PC/CMC and C-PC/CMC-CD59sp NPs were more significant for cervical cancer HeLa and SiHa cells in vitro, especially C-PC/CMC-CD59sp NPs. We found that the effect of C-PC/CMC-CD59sp NPs better than C-PC/CMC NPs is due to the advantages of CD59sp. Under the guidance of CD59sp, the targeted C-PC/CMCCD59sp NPs could specifically bind to HeLa and $\mathrm{SiHa}$ cells surface. Then the C-PC/CMC-CD59sp NPs accumulated on the HeLa and SiHa cells surface, and absorbed into cells to exert anti-cancer functions. Experimental data in vitro suggested that the anti-proliferation and pro-apoptosis effects of C-PC/CMC nanoparticles were more significant than $\mathrm{C}-\mathrm{PC}$, and further enhanced in targeted nanoparticles C-PC/CMC-CD59sp NPs.

Similarly, the C-PC/CMC and C-PC/CMCCD59sp NPs played anti-tumor roles in SiHa cells based tumor xenograft model in vivo. The growth of $\mathrm{SiHa}$ tumor xenografts was apparently suppressed in the CMC/C-PC NPs and CMC/C-PC-CD59sp NPs treatment groups. The inhibitory effects of $\mathrm{CMC} /$ C-PC-CD59sp NPs were more significant than CMC/C-PC NPs. The weights and sizes of SiHa tumor xenografts were significantly reduced in the CMC/C-PC NPs and CMC/C-PC-CD59sp NPs 
treatment groups. In addition, the tumors weights in the CMC/C-PC-CD59sp NPs treatment groups were lighter than CMC/C-PC NPs. The protein expression of apoptosis-related proteins, such as Bcl-2 and Cleaved caspase-3 had changed. The protein expression levels of Bcl-2 in the C-PC/CMC NPs and C-PC/CMC-CD59sp NPs treatment group were significantly decreased, while the expression level of Cleaved caspase-3 in the C-PC/CMC NPs and C-PC/CMC-CD59sp NPs treatment group were significantly increased. The C-PC/CMC NPs and C-PC/CMC-CD59sp NPs exerted pro-apoptotic effect by down regulating Bcl-2/Cleaved caspase-3 protein expression. The decrease of Bcl-2/Cleaved caspase-3 ratio in the C-PC/CMC-CD59sp NPs treatment group was more significant than CMC/C-PC NPs.

Simultaneously, the protein expression of cell cycle regulatory proteins involved in G1 to $S$ transition, such as Cyclin D1, CDK4 and p21 changed in cervical cancer tumor xenograft in vivo. The protein expression levels of Cyclin D1 and CDK4 in the C-PC/CMC NPs and C-PC/CMC-CD59sp NPs treatment group were significantly decreased, while the expression level of p21 in the C-PC/CMC NPs and C-PC/CMC-CD59sp NPs treatment group were significantly increased. And p21/CDK4/Cyclin D1 protein expression level changes in the C-PC/CMCCD59sp NPs treatment group were more significant than CMC/C-PC NPs. The C-PC/CMC NPs and C-PC/CMC-CD59sp NPs could up-regulate p21 proteins expression; further inhibit the expressions of Cyclin D1 and CDK4 in cervical cancer tumor xenograft in vivo.

It was reported that the MMP-2 protein acted as an angiogenic and metastatic inducers in many cancers [25]. Hence, MMPs are considered as a therapeutic target in many diseases [26]. And it was reported that C-PC selectively inhibited MMPs (MMP-2 and MMP-9) in HepG2 cells [31]. We found the MMP-2 protein expression in the C-PC/CMC NPs and C-PC/CMC-CD59sp NPs treatment group were significantly decreased, and MMP-2 protein expression level change in the C-PC/CMC-CD59sp NPs treatment group was more significant than CMC/C-PC NPs.

In short, we successfully constructed the C-PC/CMC NPs and C-PC/CMC-CD59sp NPs, and proved their anti-tumor effect in vivo and in vitro experiments in cervical cancer HeLa and SiHa cells (Figure 9). We proved that the anti-tumor effects of C-PC/CMC-CD59sp NPs were better than C-PC and C-PC/CMC nanoparticles on cervical cancer due to the targeting effect of CD59sp in vitro and in vivo.

\section{Abbreviations}

C-PC: C-phycocyanin; CMC: Carboxymethyl chitosan; NPs: nanoparticle; CD59sp: CD59- specific ligand peptide.

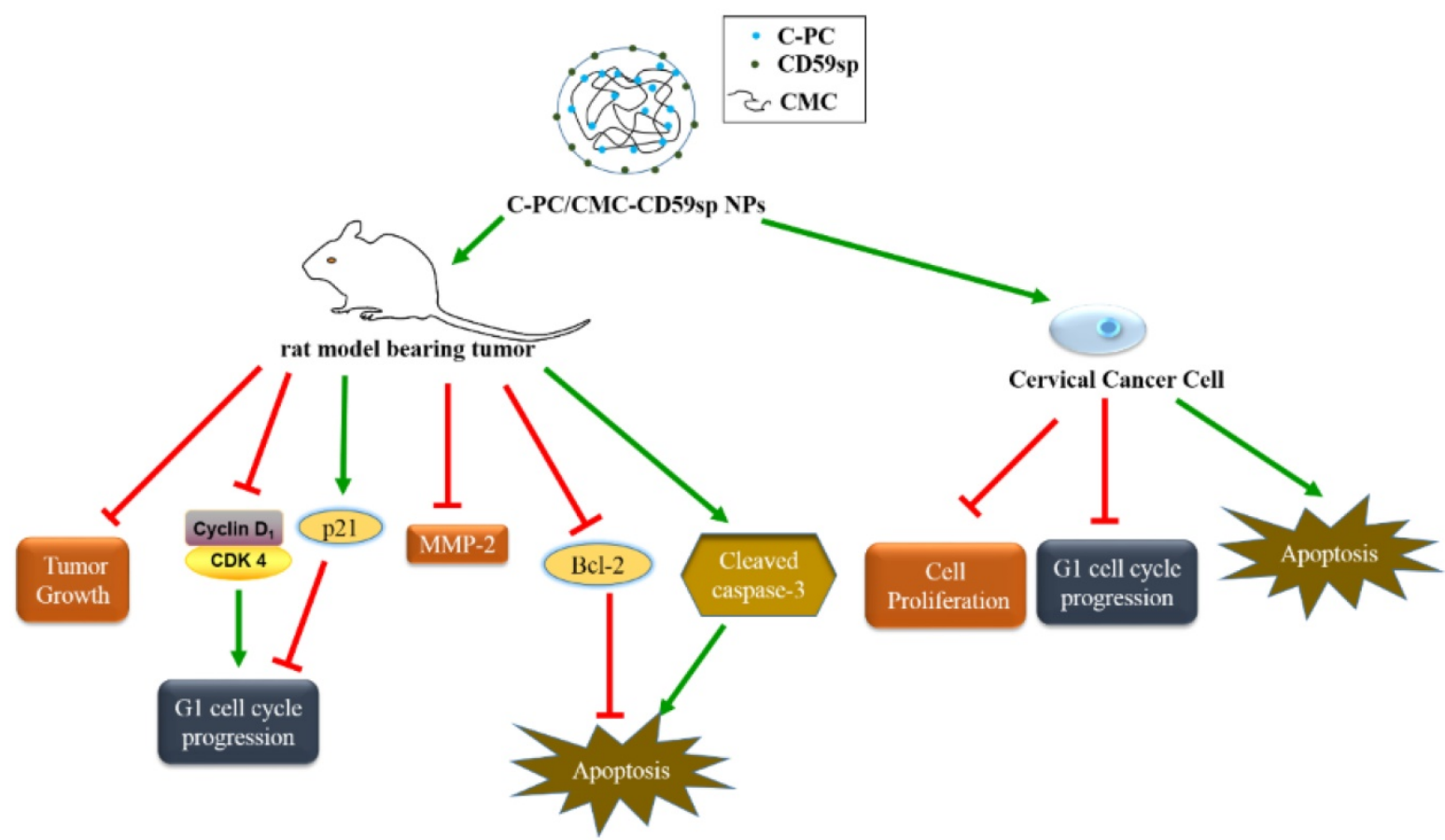

Figure 9. Schematic model of the proposed anti-cancer mechanisms for C-PC/CMC-CD59sp NPs in cervical cancer. 


\section{Acknowledgments}

This work was supported by grants from the National Natural Science Foundation of China (81471546, 81001346, 81273206).

\section{Competing Interests}

The authors have declared that no competing interest exists.

\section{References}

1. Torre LA, Bray F, Siegel RL, Ferlay J, Lortet-Tieulent J, Jemal A. Global cancer statistics, 2012. CA: a cancer journal for clinicians. 2015; 65: 87-108.

2. Siegel RL, Miller KD, Jemal A. Cancer Statistics, 2017. CA: a cancer journal for clinicians. 2017; 67: 7-30.

3. Wang Y, Jiang L, Yin Q, Liu H, Liu G, Zhu G, et al. The Targeted Antitumor Effects of C- PC/CMC-CD59sp Nanoparticles on HeLa Cells in Vitro and in Vivo. Journal of Cancer. 2017; 8: 3001-13.

4. Jiang L, Wang Y, Yin Q, Liu G, Liu H, Huang Y, et al. Phycocyanin: A Potential Drug for Cancer Treatment. Journal of Cancer. 2017; 8: 3416-29.

5. Liu Q, Huang Y, Zhang R, Cai T, Cai Y. Medical Application of Spirulina platensis Derived C-Phycocyanin. Evidence-based complementary and alternative medicine : eCAM. 2016; 2016: 7803846.

6. Davis ME, Chen ZG, Shin DM. Nanoparticle therapeutics: an emerging treatment modality for cancer. Nature reviews Drug discovery. 2008; 7: 771-82.

7. Jayakumar R, Prabaharan M, Nair SV, Tokura S, Tamura H, Selvamurugan N. Novel carboxymethyl derivatives of chitin and chitosan materials and their biomedical applications. Prog Mater Sci. 2010; 55: 675-709.

8. Wang Y, Qin F, Tan H, Zhang Y, Jiang M, Lu M, et al. pH-responsive glycol chitosan-cross-linked carboxymethyl-beta-cyclodextrin nanoparticles for controlled release of anticancer drugs. International journal of nanomedicine. 2015; 10: 7359-69.

9. Vasir JK, Labhasetwar V. Targeted drug delivery in cancer therapy. Technology in cancer research \& treatment. 2005; 4: 363-74.

10. Costello RT, Gastaut JA, Olive D. What is the real role of CD40 in cancer immunotherapy? Immunology today. 1999; 20: 488-93.

11. Henson E, Chen Y, Gibson S. EGFR Family Members' Regulation of Autophagy Is at a Crossroads of Cell Survival and Death in Cancer. Cancers. 2017; 9 .

12. Day KC, Lorenzatti Hiles G, Kozminsky M, Dawsey SJ, Paul A, Broses LJ, et al. HER2 and EGFR Overexpression Support Metastatic Progression of Prostate Cancer to Bone. Cancer research. 2017; 77: 74-85.

13. Pandey S, Mahtab A, Rai N, Rawat P, Ahmad FJ, Talegaonkar S. Emerging Role of CD44 Receptor as a Potential Target in Disease Diagnosis: A Patent Review. Recent patents on inflammation \& allergy drug discovery. 2017; 11: 77-91.

14. Gasparetto M, Smith CA. ALDHs in normal and malignant hematopoietic cells: Potential new avenues for treatment of AML and other blood cancers. Chemico-biological interactions. 2017; 276: 46-51.

15. Watson NF, Durrant LG, Madjd Z, Ellis IO, Scholefield JH, Spendlove I. Expression of the membrane complement regulatory protein CD59 (protectin) is associated with reduced survival in colorectal cancer patients. Cancer immunology, immunotherapy : CII. 2006; 55: 973-80.

16. Varsano S, Rashkovsky L, Shapiro H, Ophir D, Mark-Bentankur T. Human lung cancer cell lines express cell membrane complement inhibitory proteins and are extremely resistant to complement-mediated lysis; a comparison with normal human respiratory epithelium in vitro, and an insight into mechanism(s) of resistance. Clinical and experimental immunology. 1998; 113: 173-82.

17. Crnogorac-Jurcevic T, Efthimiou E, Nielsen T, Loader J, Terris B, Stamp G, et al. Expression profiling of microdissected pancreatic adenocarcinomas. Oncogene. 2002; 21: 4587-94.

18. Fishelson Z, Donin N, Zell S, Schultz S, Kirschfink M. Obstacles to cancer immunotherapy: expression of membrane complement regulatory proteins (mCRPs) in tumors. Molecular immunology. 2003; 40: 109-23.

19. Afshar-Kharghan V. The role of the complement system in cancer. The Journal of clinical investigation. 2017; 127: 780-9.

20. Li B, Gao MH, Chu XM, Xu YJ, Yang F. Identification of a novel short peptide seal specific to CD59 and its effect on HeLa cell growth and apoptosis. Cellular oncology. 2012; 35: 355-65.

21. Lin A, Chen J, Liu Y, Deng S, Wu Z, Huang Y, et al. Preparation and evaluation of $\mathrm{N}$-caproyl chitosan nanoparticles surface modified with glycyrrhizin for hepatocyte targeting. Drug development and industrial pharmacy. 2009; 35: 1348-55.

22. Anandhakumar S, Krishnamoorthy G, Ramkumar KM, Raichur AM. Preparation of collagen peptide functionalized chitosan nanoparticles by ionic gelation method: An effective carrier system for encapsulation and release of doxorubicin for cancer drug delivery. Materials science \& engineering C, Materials for biological applications. 2017; 70: 378-85.
23. Yang P, Li B, Yin QF, Wang YJ. Carboxymethyl chitosan nanoparticles coupled with CD59-specific ligand peptide for targeted delivery of C-phycocyanin to HeLa cells. Tumour biology : the journal of the International Society for Oncodevelopmental Biology and Medicine. 2017; 39: 1010428317692267.

24. Sherr CJ, Roberts JM. CDK inhibitors: positive and negative regulators of G1-phase progression. Genes \& development. 1999; 13: 1501-12.

25. Milia-Argeiti E, Huet E, Labropoulou VT, Mourah S, Fenichel P, Karamanos NK, et al. Imbalance of MMP-2 and MMP-9 expression versus TIMP-1 and TIMP-2 reflects increased invasiveness of human testicular germ cell tumours. International journal of andrology. 2012; 35: 835-44.

26. Radisky ES, Radisky DC. Matrix metalloproteinases as breast cancer drivers and therapeutic targets. Frontiers in bioscience. 2015; 20: 1144-63.

27. Small W, Jr., Bacon MA, Bajaj A, Chuang LT, Fisher BJ, Harkenrider MM, et al. Cervical cancer: A global health crisis. Cancer. 2017; 123: 2404-12.

28. Ferlay J, Soerjomataram I, Dikshit R, Eser S, Mathers C, Rebelo M, et al. Cancer incidence and mortality worldwide: sources, methods and major patterns in GLOBOCAN 2012. International journal of cancer. 2015; 136: E359-86.

29. Pereira E, Cooper HH, Zelaya PG, Creasman W, Price FV, Gupta V, et al. Concurrent chemoradiation versus radiotherapy alone for the treatment of locally advanced cervical cancer in a low-resource setting. Gynecologic oncology reports. 2017; 19: 50-2.

30. Bermudez A, Bhatla N, Leung E. Cancer of the cervix uteri. International journal of gynaecology and obstetrics: the official organ of the International Federation of Gynaecology and Obstetrics. 2015; 131 Suppl 2: S88-95.

31. Kunte M, Desai K. The Inhibitory Effect of C-phycocyanin Containing Protein Extract (C-PC Extract) on Human Matrix Metalloproteinases (MMP-2 and MMP-9) in Hepatocellular Cancer Cell Line (HepG2). The protein journal. 2017; 36: 186-95. 